\title{
PROMIS Adult Physical Health Domain
}

National Cancer Institute

\section{Source}

National Cancer Institute. PROMIS Adult Physical Health Domain. NCI Thesaurus. Code C122346.

A Patient Reported Outcomes Measurement Information System (PROMIS) domain that assesses the status of an individual's physical health. 